\title{
Einleitung: Neue Perspektiven auf das gute Leben
}

Die Frage, was ein gutes Leben ist, dürfte wohl jedem von uns vertraut sein. Wir stellen sie zumeist in praktischer Absicht: Wir fragen danach, wie wir unser Leben alles in allem betrachtet führen sollen. Zweifellos steht sie nicht im Zentrum des alltäglichen Denkens. Wir beschäftigen uns mehr mit anderen Dingen wie dem alltäglichen Einkauf, dem Berufsalltag und den Sportergebnissen. Dies ist auch nicht überraschend: Die kulturelle Gemeinschaft, in die wir hineingeboren werden, stellt nämlich bereits zahlreiche Muster für unsere Lebensführung bereit. Wir erlernen solche Leitmotive, wie zu leben gut ist, bereits in der Kindheit. Dementsprechend müssen wir uns die Frage nach dem guten Leben auch nicht ständig vorhalten. Dennoch ist nicht von der Hand zu weisen, dass sie von Zeit zu Zeit über uns hereinbricht. Wir hegen mitunter Zweifel, ob die überlieferten Deutungsmuster auch die richtigen Maßstäbe darstellen, an denen wir uns orientieren sollen. Besonders nachdrücklich stellt sich dieser Zweifel etwa in historischen Zeiten ein, die selbst durch Wertumbrüche und Krisen gekennzeichnet sind. Wir sind uns in diesen Momenten nicht mehr sicher, wie wir unser Leben eigentlich führen sollen, und suchen daher nach möglicher Orientierung.

Doch wen können wir diesbezüglich um Rat fragen? In der Antike war die Ansicht verbreitet, dass es vor allem die Philosophie sei, die etwas zur Thematik des guten Lebens zu sagen habe. ${ }^{1}$ Diese Ansicht wurde allerdings in der Neuzeit erheblich in Zweifel gezogen. War es früher die Philosophie, die uns über die richtige Lebensführung aufklärte, sind es nun die empirischen Sozialwissenschaften, denen zusammen mit populärwissenschaftlicher Literatur zum glücklichen Leben ein „Erstzugriffsrecht“ eingeräumt wird. Es ist viel über die ideengeschichtlichen Ursachen dieser Entwicklung und die Frage, ob man sie gutheißen sollte oder nicht, geschrieben worden. ${ }^{2}$ Aus historischer Sicht betrachtet ist jedoch wichtiger, dass mit dem so gearteten Primat nicht das letzte Wort gesprochen ist. Denn in der jüngeren Vergangenheit zeichnet sich die Tendenz ab, dass sich die Philosophie schrittweise dem Thema wieder zuwendet. Aussagekräftiger Indikator für ein solches Comeback ist beispielsweise die philosophische Auseinandersetzung mit der Thematik in der analytischen Ethik der 80er und 90er

1 Vgl. dafür die Rekonstruktion in Horn 1998 und Wolf 1999.

2 Eine ausführliche Beschreibung der „Verfallsgeschichte“ und ihrer ambivalenten Bewertungen findet sich etwa in Fenner 2007, Kap. 2. 
Jahre. Hier haben insbesondere die Arbeiten von Alasdair MacIntyre, Martha Nussbaum und Charles Taylor, um einmal drei prominente Namen herauszugreifen, eine breite Rezeption erfahren und so maßgeblich zum Wiedererwachen des philosophischen Interesses an einer Theorie des guten Lebens beigetragen. ${ }^{3} \mathrm{Es}$ scheint daher nur konsequent, wenn einige Autoren von einer Renaissance des guten Lebens als eines der Kernthemen der Philosophie sprechen. ${ }^{4}$ In der Gegenwart hat sich dieses Interesse noch verstärkt. So verrät ein Blick auf die einschlägige Fachliteratur, dass in den letzten Jahren ein respektabler Fundus an weiteren Texten entstanden ist. Es gibt mittlerweile eine ganze Reihe von Einführungsbüchern zum Thema; ${ }^{5}$ eine kaum noch überschaubare Vielfalt von Einzelmonographien; ${ }^{6}$ sowie mehrere neuere Aufsatzsammlungen, ${ }^{7}$ in denen sich Denker mit der Frage nach dem guten Leben auseinandersetzen.

Hierbei handelt es sich aber nicht nur um eine quantitative Fortentwicklung. Vielmehr zeigt ein genauer Blick auf das Forschungsfeld, dass auch eine gewachsene Heterogenität der Fragestellungen zu beobachten ist. Während zu Beginn der Debatte die Frage nach dem guten Leben vorwiegend auf die Metaethik begrenzt war, findet man sie mittlerweile in ganz verschiedenen philosophischen Bereichen wieder. ${ }^{8}$ Man fragt nicht mehr etwa nur nach den begrifflichen $\mathrm{Zu}-$ sammenhängen von Glück und gutem Leben, sondern beispielsweise auch nach der Verbindung zwischen gutem Leben und sozialer Gerechtigkeit. Brauchen wir eine Vorstellung eines gelungenen Lebens, um gerechte von ungerechten Güterverteilungen zu unterscheiden? Und wenn ja, welche könnte dies sein? - Diese und weitere Fragen stellen sich in der politischen Philosophie. ${ }^{9}$ Weiterhin: Wie hängen Begriffe wie „lebensunwertes Leben“ und „würdiges Sterben“ mit dem Begriff des „guten Lebens“ zusammen? Können wir aus unseren Vorstellungen über Letzteres nicht auch Leitlinien für die ersten beiden Begriffe gewinnen? Welche konkreten Inhalte könnten dies sein? - Dies sind Fragen, die unter anderem in den Bereich

3 Vgl. Maclntyre 1981, Taylor 1989, Nussbaum 1992.

4 Vgl. Bien 1995, Seel 1995 und Fenner 2007, 7.

5 Vgl. etwa Fenner 2007 und Kazez 2007.

6 Um nur einige einschlägige Titel herauszugreifen: Crisp 2006, Haybron 2008, Kraut 2008, Tiberius 2008, Russell 2009, Bradley 2011 und Wessels 2011.

7 Claussen 2005, Gardiner 2005, Chappell 2006 und Olsaretti 2006.

8 Diese metaethische „Stoßrichtung“ wird etwa in den Beiträgen des einschlägigen Sammelbandes von Holmer Steinfath ausführlich reflektiert. Vgl. Steinfath 1998.

9 Vgl. dazu etwa Nussbaum 2006, die die Frage nach dem guten Leben explizit im Zusammenhang mit einer Theorie der Gerechtigkeit diskutiert. 
der angewandten Ethik fallen. ${ }^{10}$ Und schließlich: Verweisen unsere Ideen von einem guten Leben nicht in letzter Instanz auf ein höheres Wesen? Ist nicht ohne die Annahme eines göttlichen Schöpfers alles gleichgültig? Wie hängen die Kategorien „Sinn“ und „gutes Leben“ zusammen? - Dies sind Fragen, die mitunter dem Einzugsbereich der Religionsphilosophie zugerechnet werden können. ${ }^{11}$

Sicherlich ließen sich noch weitere Diskussionsfelder aufzählen. Diese kurze Aufstellung sollte aber bereits ausreichen, um den wesentlichen Punkt deutlich zu machen: Es ist eine neue Forschungssituation eingetreten. Im Vergleich zu den Anfängen zeichnet sich die gegenwärtige philosophische Auseinandersetzung mit dem guten Leben durch eine thematische Breite aus, die bisher in diesem Maße nicht anzutreffen war. Das gute Leben wird nicht nur im Kontext der Metaethik verhandelt, sondern spielt auch für Fragestellungen der politischen Philosophie, der angewandten Ethik und der Religionsphilosophie eine bedeutende Rolle.

Diese Beobachtung bildet den Ausgangspunkt des vorliegenden Sammelbandes. Er ist in der Absicht entstanden, die neue Vielfalt an Fragen, die unter dem Stichwort „gutes Leben“ zusammengefasst werden, möglichst breit und unter Einbeziehung der neuesten Entwicklungen abzudecken. Die behandelten Themen reichen von der klassischen metaethischen Gegenüberstellung subjektiver und objektiver Theorien des guten Lebens über den Glücksbegriff, das Verhältnis von Tugend und Glück sowie die Perfektionismus- und die Liberalismusdebatten bis hin zur Frage nach dem Sinn des Lebens. Ein Anspruch auf Vollständigkeit kann damit selbstredend nicht verbunden sein. Der gesamte Bereich der angewandten Ethik sowie Gerechtigkeitsfragen im engeren Sinn mussten ausgeklammert bleiben.

Ein besonderes Augenmerk wird auf das Verhältnis philosophischer und theologischer bzw. religionsphilosophischer Ansätze gelegt. Auch über diejenigen Beiträge hinaus, die sich explizit dieser Frage widmen, rückt sie mehrfach ins Blickfeld. Diese dezente Schwerpunktsetzung ist der Tatsache geschuldet, dass ein großer Teil der Beiträge des Bandes auf ein Symposium mit dem Titel Philosophie, Theologie und die Frage nach dem guten Leben zurückgeht, das im Juni 2011 in Münster stattfand. Die Konfrontation der wiederauflebenden philosophischen

10 Dabei wird die Frage nach dem guten Leben in ganz verschiedenen Problemkontexten der angewandten Ethik diskutiert. Siehe für eine Auswahl an verschiedenen Zusammenhängen Bragues 2006, George 2008, Buchanan 2009 und Johnson 2010.

11 Siehe hierzu etwa Cottingham 2005, Claussen 2005; Miggelbrink 2009 und Disse/Goebel 2010. Neu ist dabei, dass sich die von philosophischer Seite betriebene Religionsphilosophie und die theologische Debatte treffen. Dass dagegen die Theologie selbst der Frage nach dem guten Leben wenigstens implizit immer schon eine zentrale Stellung eingeräumt hat, ist eine historische Beobachtung, die weitgehend unstrittig ist. 
Ansätze mit der Theologie verspricht in mehreren Hinsichten eine lebhafte und produktive Auseinandersetzung: Nicht nur hat die Theologie im Gegensatz zur säkularen Denktradition seit ihren Anfängen an einem „verbindliche[n] Begriff vom guten und exemplarischen Leben“ festgehalten und „Intuitionen von Verfehlung und Erlösung, vom rettenden Ausgang aus einem als heillos erfahrenen Leben artikuliert, über Jahrtausende hinweg subtil ausbuchstabiert und hermeneutisch wachgehalten““ ${ }^{12}$ Sie tendiert auch zu metaphysischen Annahmen, die vielen als notwendig erscheinen, um substantiellen Aussagen über das gute Leben ein Fundament zu geben. $\mathrm{Zu}$ guter Letzt wird die Theologie im akademischen wie außerakademischen Kontext so eng mit der Frage nach dem Sinn des Lebens (oder gar allgemein mit Sinnfragen) verknüpft, dass für viele eine jede Sinnfrage als eine im weitesten Sinne religiöse Frage aufzufassen ist. ${ }^{13}$ Insofern Sinn ein Bestandteil des guten Lebens ist, scheint es daher mindestens plausibel zu sein, dass die Frage nach dem guten Leben im Grenzbereich von Philosophie und Theologie zu situieren ist.

Dieser doppelten Motivation folgend sollen zunächst in einem ersten Teil die theoretischen Grundlagenfragen diskutiert werden, die sich stellen, wenn danach gefragt wird, was ein gutes Leben ist.

In diesem Zusammenhang fragt Holmer Steinfath zunächst nach den zentralen Begriffen und Wertdimensionen, die bei der Beschäftigung mit der Thematik des guten Lebens eine Rolle spielen müssen. Steinfath betont die Notwendigkeit starker Wertungen bei der Bestimmung des guten Lebens; nur so lasse sich die von diesem in Anspruch genommene Orientierungsfunktion erhalten. Derlei „starke Wertungen“ wiederum benötigten zum einen eine emotionale Anbindung an unser Wünschen und Wollen und müssten sich zweitens einer intersubjektiven Prüfung unterziehen, um hinreichende Stabilität und Nutzbarkeit für unser Leben entwickeln zu können.

Kurt Bayertz stellt demgegenüber methodische Überlegungen in den Vordergrund: Er fragt nach der Rolle empirischer Erkenntnisse bei der Bestimmung des guten Lebens. Im Ergebnis erhalten empirische Methoden und ihre Resultate im Rahmen der sogenannten Glücksforschung ein gewichtiges Mitspracherecht innerhalb der philosophischen Diskussion des guten Lebens zugesprochen. Allerdings lassen sich auch Bereiche identifizieren, in denen die Philosophie ein gutes Wort mitzureden hat, was etwa die begrifflichen Grundlagen der empirischen Forschungen angeht.

12 Habermas 2005, 31.

13 In der von Niklas Luhmann begründeten funktionalen Bestimmung des Religionsbegriffs wird dieser bekanntlich durch den Bezug zur Sinnfrage definiert. 
Die beiden folgenden Beiträge beschäftigen sich mit den ontologischen Grundannahmen, die eine plausible Theorie des guten Lebens akzeptieren muss.

Markus Rüther wägt die Vor- und Nachteile einer objektivistischen Konzeption des guten Lebens gegenüber ihren Alternativen im Sinne eines Forschungsplans gegeneinander ab. Im Ergebnis sieht er die Beweislast, jedenfalls was den Ausgangspunkt der Debatte betrifft, eher bei subjektivistischen Fassungen des guten Lebens: Die weit verbreitete Skepsis, die ihren objektivistischen Mitbewerbern entgegen schlägt, erweist sich weder als aus der metatheoretischen Perspektive begründet, was etwa das Verhältnis von Theorie und Alltagspraxis angeht; noch kann mit Bezug auf die konkreten inhaltlichen Fragen dem Objektivismus der Vorwurf gemacht werden, er würde der individuellen Normativität einen zu geringen Spielraum einräumen.

Peter Schaber argumentiert darüber hinausgehend dezidiert für eine objektivistische Konzeption. Er begründet dies mit der Alternativlosigkeit einer objektivistischen Basis zur Rechtfertigung unserer subjektiven Einstellungen, die andernfalls für ethische Fragestellungen unbewertbar blieben. Menschen können sich demnach sehr wohl darüber irren, was für sie gut ist, auch wenn es sich um höchstpersönliche Eigenschaften handelt wie diejenige, was sie selbst sind oder sein können. Dementsprechend muss dasjenige, was Menschen faktisch für gut halten, nicht das sein, was gut für sie ist. Eben dies motiviert für Schaber die Frage nach dem guten Leben.

Der zweite Teil des Bandes setzt die Beschäftigung mit den theoretischen Grundlagen des behandelten Themas fort, nun allerdings im Sinne einer $A b$ grenzung von verwandten Begriffen, die in der Debatte um das gute Leben eine Rolle spielen.

Den Anfang macht Ludwig Siep, der sich mit dem zum guten Leben häufig synonym gebrauchten Begriff des sinnvollen Lebens auseinandersetzt und hier unterschiedliche Lesarten voneinander trennt. Ihm geht es zentral darum, die Möglichkeit von Theorien des sinnvollen Lebens, hinreichend stabile Kriterien zur Bestimmung ihres Sujets zu entwickeln, einzuschätzen. Siep kritisiert die Beschränkung der Sinnfrage auf den (einen, umfassenden, letzten) Sinn des Lebens und betont die Pluralität von Kriterien und Weisen sinnvollen Lebens. Sie sind nicht auf die Annahme oder Suche nach einer transzendenten Sinnquelle angewiesen. Gleichwohl gehören auch solche Annahmen zu den möglichen Antworten auf die Frage nach einem sinnvollen Leben.

Thaddeus Metz nimmt diesen Gedanken auf und fragt nach der philosophischen Differenz zwischen sinnvollem und lebenswertem Leben, um in Folge ihre jeweilige Relevanz zur Charakterisierung des guten Lebens herauszuarbeiten. Beide können im Grundsatz unabhängig voneinander bestehen, wenngleich sie im Einzelfall meist kontingenterweise zusammenfallen. In diesem Fall werden und 
sollten beide Wertdimensionen unverzichtbare Ingredienzen eines gelungenen im Sinne eines guten Lebens sein.

Peter Strasser richtet sein Interesse auf die Unterscheidung zwischen dem Ideal eines guten Lebens und den weitaus niedriger anzusetzenden minimalen Anforderungen, ein solches auch tatsächlich zu führen. Ersteres wird von vielen Philosophen, gerade wenn man es in objektivistischer Art und Weise ausbuchstabiert, als zu „abgehoben“ gegenüber dem konkret zu führenden Leben empfunden. Dagegen argumentiert Strasser, dass ein solches Idealbild geradezu notwendig ist, da es die unverzichtbare Grundlage einer jeden menschlichen Existenz bildet, die danach strebt, sich im Rückblick mit sich selbst auszusöhnen: Am Ende mag ich mein unvolkommenes Leben, vor dem Hintergrund meines geringen Anteils am guten Leben, immerhin als „gut genug“ erkennen.

Christoph Halbig schließlich greift die von Metz angesprochene Dimension des lebenswerten oder glücklichen Lebens erneut auf und untersucht diese mit Blick auf eine weitere, eng mit dem guten Leben zusammenhängenden Wertdimension, dem moralischen Leben. Die Unterscheidung verschiedener Ebenen, auf denen sich die Frage nach dem Verhältnis von Tugend und Glück stellt, führt zu einem vielschichtigen Ergebnis: Zwar kann weder ein tugendhaftes Leben ein glückliches Leben garantieren, noch muss sich ein glückliches Leben notwendigerweise durch Tugendhaftigkeit auszeichnen. Dennoch stehen in mehrfacher Hinsicht Tugend und Glück in einer Wechselbeziehung, was sich zum Beispiel darin zeigt, dass bestimmte Tugenden eine konstitutive Rolle für prudentielle Güter haben.

Innerhalb der Debatte um das gute Leben dürfte der Perfektionismus mit seiner Leitidee einer Vervollkommnung menschlicher Anlagen mit die bedeutendste Spielart objektivistischer Theorien darstellen. Um diese Theoriefamilie kreisen die Beiträge des dritten Teils des Bandes.

Franz-Josef Bormann macht sich für einen schwachen Perfektionismus stark, demzufolge ein allgemeines und abstraktes Kriterium der Lebensführung mit einer Pluralität von Lebensformen verträglich sei. Nach einer kritischen Diskussion der Theorien A. MacIntyres und Ph. Foots begründet er dies mit einer an den USamerikanischen Philosophen A. Gewirth angelehnten Theorie der Handlungsfähigkeit. Demnach sei aufgrund der Unhintergehbarkeit menschlichen Handelns eine jede Konzeption des guten Lebens der Bedingung unterworfen, die Vervollkommnung der eigenen Handlungsfähigkeit ebenso wie die der Handlungsfähigkeit anderer zu gewährleisten.

Perfektionistische Theorien haben zumindest die Tendenz, den Wert autonomer Entscheidungen nur schwer integrieren zu können; kann doch das Ergebnis solcher Entscheidungen der Vervollkommnung des Menschen gerade entgegenstehen. Sebastian Muders untersucht deshalb Bemühungen von Seiten antiper- 
fektionistisch eingestellter, liberaler Theorien, eine den objektivistischen Intuitionen angemessene Theorie des guten Lebens mit einer starken Betonung des Wertes personaler Autonomie $\mathrm{zu}$ verbinden. Insbesondere werde eine solche Verbindung durch eine Kopplung der liberalen Theorie mit tugendethischen Ansätzen versucht, was allerdings zu ernsthaften Spannungen führe, die diese Theorien leicht dem Vorwurf des Relativismus aussetzten. Im Ergebnis argumentiert Muders deshalb für eine nur schwache Berücksichtigung des Werts autonomer Entscheidungen. Diese reiche auch aus, die ebenfalls vorhandenen subjektivistischen Intuitionen, welche die Frage nach der guten Lebensführung als höchstpersönliche Angelegenheit betrachten, zu befriedigen.

Matthias Hoesch versucht schließlich, die Diskussion um das gute Leben mit der Debatte um die Existenz von Pflichten gegen sich selbst zu verknüpfen. Er sieht dabei einen engen Zusammenhang zwischen beiden Themen: Wer die Existenz von Pflichten gegen sich selbst bejaht, sei wenigstens in einem bestimmten Sinn auf eine objektive Theorie des guten Lebens festgelegt; wer eine objektive Theorie des guten Lebens vertritt, müsse andersherum auch die Existenz von Pflichten gegen sich selbst zugestehen. Hoesch setzt sich in diesem Zusammenhang insbesondere mit dem Vorwurf auseinander, dass Pflichten gegen sich selbst einen selbstwidersprüchlichen Begriff bildeten.

Der vierte und letzte Teil des Bandes beschäftigt sich mit dem Problem, inwieweit eine objektivistische Theorie des guten Lebens auf das Transzendente angewiesen ist, und wie sich dies aus philosophischer wie theologischer Perspektive charakterisieren lässt.

Jörg Disse argumentiert für eine enge Verbindung zwischen objektivistischen und theistischen Theorien des guten Lebens: Das gute Leben für den Atheisten könne nur in der Verfolgung seines eigenen Glücks in Form subjektiver Interessen bestehen; das gute Leben für den Theisten umfasse hingegen darüber hinaus die universale Verwirklichung des Guten um seiner selbst willen. Disse begründet diese These mit einer Theorie menschlicher Zielsetzungen, die an Ansätze der empirischen Kognitionspsychologie anknüpft. Folgt man dieser Theorie, lasse nur der Glaube an eine künftige vollkommene Gerechtigkeit die Verwirklichung des Guten um seiner selbst willen als sinnvolles Ziel menschlichen Handelns erscheinen.

John Cottinghams Überlegungen nehmen ihren Ausgangspunkt in einem transzendenten Bedürfnis des Menschen, das tief in seiner Natur verwurzelt sei. Cottingham illustriert dieses Bedürfnis in drei Dimensionen, einer kosmologischen, einer ästhetischen und einer moralischen. Er argumentiert gegen naturalistisch inspirierte Versuche, das Bedürfnis nach dem Transzendenten deflationistisch oder reduktiv zu behandeln, und kommt $\mathrm{zu}$ dem Ergebnis, dass der Theismus, wie er etwa in der Abrahamitischen Religionen vorliegt, eine 
adäquatere Antwort darstelle. Als Folge dieses ,phänomenologischen Arguments läge es nahe, die Befriedigung des transzendenten Bedürfnisses als notwendigen Bestandteil des guten Lebens anzusehen.

Während die Argumentationen von Cottingham und Disse eher der natürlichen Theologie oder Religionsphilosophie zuzuordnen sind, nähert sich Sigrid Müller im abschließenden Beitrag der Frage nach dem guten Leben aus einem distinkt christlich-theologischen Blickwinkel. Wie die Philosophie habe auch die Theologie im 20. Jahrhundert eine „Wende zum Subjekt“ vollzogen. Obwohl damit etwa ein von kirchlichen Autoritäten vorgegebener Katalog von Normen zur Lebensgestaltung unmöglich geworden sei, versucht Müller, einige Aspekte ausfindig zu machen, die für die christliche Vorstellung eines guten Lebens besonders charakteristisch sind. Die Hoffnung auf Auferstehung und Erlösung führten demnach zu einem Begriff der Lebensfülle, der auch Scheitern und Fehlbarkeit integrieren könne.

Das Projekt eines Sammelbandes, wie er nun fertig vorliegt, lebt selbstverständlich von der Mitwirkung und Unterstützung vieler. Für die finanzielle Beteiligung, die uns sowohl das Symposium Philosophie, Theologie und die Frage nach dem guten Leben als auch den vorliegenden Band erst ermöglichte, bedanken wir uns bei der Bischöflichen Studienförderung Cusanuswerk sowie bei der Graduiertenschule des Exzellenzclusters „Religion und Politik“. Unserem gemeinsamen Doktorvater Ludwig Siep sind wir in vielerlei Hinsicht zu großen Dank verpflichtet; er unterstützte uns in jeder Phase des Projekts mit Rat und Tat. Dank sei auch allen Teilnehmern und Vortragenden des Symposiums ausgesprochen, deren lebhafte Diskussionsbeiträge vielfach Eingang in den Sammelband gefunden haben, sowie der Lektorin Gertrud Grünkorn vom Verlag Walter de Gruyter, die für uns bei der Konzeption und Organisation des Bandes eine unerlässliche Ansprechpartnerin war. Der größte Dank gilt freilich allen Beitragenden, mit deren Kompetenz, Zeit und Engagement sich das Herzstück des Sammelbands, die philosophische Auseinandersetzung mit dem guten Leben, nur realisieren ließ.

\section{Literatur}

Bien (1995): Günther Bien, „Über das Glück“, Information Philosophie 1, 5-16.

Bradley (2011): Ben Bradley, Well-Being and Death, Oxford.

Bragues (2006): George Brague, „Seek the Good Life, not Money: The Aristotelian Approach to

Business Ethics“, Journal of Business Ethics 67(4), 341-357.

Buchanan (2009): Alan Buchanan, „Human Nature and Enhancement“, Bioethics 32(3),

$141-150$.

Chappell (2006): Timothy Chappell (Hg.), Virtues and Values, Oxford. 
Claussen (2005): Johann Claussen (Hg.), Glück und Gegenglück: Philosophische und theologische Variationen zu einem alltäglichen Begriff, Tübingen.

Crisp (2006): Roger Crisp, Reasons and the Good, Oxford.

Cottingham (2005): John Cottingham, The Spiritual Dimension: Religion, Philosophy and Human Values, Cambridge.

Disse/Goebel (2010): Jörg Disse und Bernd Goebel (Hg.), Gott und die Frage nach dem Glück. Anthropologische und ethische Perspektiven, Frankfurt a. M.

Fenner (2007): Dagmar Fenner, Das gute Leben, Berlin.

George (2008): Robert George, „The Nature and Basis of Human Dignity“, Ratio Juris 21(2), $173-193$.

Habermas (2005): Jürgen Habermas, „Vorpolitische Grundlagen des demokratischen Rechtsstaates?“, in: Florian Schuller (Hg.), Dialektik der Säkularisierung, Freiburg i. Br., $15-37$.

Haybron (2008): Daniel M. Haybron, The Pursuit of Unhappiness, Oxford.

Horn (1999): Christoph Horn, Antike Lebenskunst, München.

Kraut (2008): Richard Kraut, What is Good and Why, Cambridge.

Gardiner (2005): Stephen Gardiner (Hg.), Virtue Ethics. Old and New, New York.

Johnson (2010): Lawrence Johnson, A Life-Centered Approach to Bioethics, Cambridge.

Kazez (2007): Jean Kazez, The Weight of Things: Philosophy and the Good Life, Oxford.

Maylntre (1981): Alasdair MacIntyre, After Virtue, Notre Dame.

Miggelbrink (2009): Ralf Miggelbrink, Lebensfülle. Für die Wiederentdeckung einer theologischen Kategorie, Freiburg i. Br. u.a.

Nussbaum (1992): Martha Nussbaum, „Human Functioning and Social Justice. In Defense of Aristotelian Essentialism“, Political Theory 20/2, 202-246.

Nussbaum (2006): Martha Nussbaum, Frontiers of Justice, Cambridge.

Olsaretti (2006): Serena Olsaretti (Hg.), Preferences and Well-Being, Cambridge.

Russell (2009): Daniel Russell: Practical Intelligence and the Virtues, Oxford.

Seel (1995): Martin Seel, Versuch über die Formen des Glücks, Frankfurt a. M.

Steinfath (1998): Holmer Steinfath (Hg.), Was ist ein gutes Leben? Philosophische Reflexionen, Frankfurt a. M.

Taylor (1989): Charles Taylor, Sources of the Self, Cambridge.

Tiberius (2008): Valerie Tiberius, The Reflective Life. Living Wisely with Our Limits, Oxford.

Wessels (2011): Ulla Wessels, Das Gute, Frankfurt a. M.

Wolf (1999): Ursula Wolf, Philosophie und die Frage nach dem guten Leben, Hamburg. 